\title{
Peripapillary choroidal thickness by enhanced depth imaging optical coherence tomography: the impact of metabolic syndrome
}

\author{
Mónica Loureiro ${ }^{1,2}$, Ana Cristina Braga ${ }^{3}$, Dália Meira ${ }^{1}$, Paula Sepúlveda ${ }^{1}$, Luís Agrelos ${ }^{1}$, Paulo Torres ${ }^{2,4}$ \\ ${ }^{1}$ Department of Ophthalmology, Centro Hospitalar de V. N. Gaia/Espinho, Vila Nova de Gaia - Portugal \\ ${ }^{2}$ Institute of Biomedical Sciences Abel Salazar, University of Porto, Porto - Portugal \\ ${ }^{3}$ ALGORITMI Centre, University of Minho, Braga - Portugal \\ ${ }^{4}$ Department of Ophthalmology, Centro Hospitalar do Porto, Porto - Portugal
}

\begin{abstract}
Purpose: To assess the impact of metabolic syndrome (MetS) on the peripapillary choroidal thickness (PPCT) and to characterize the PPCT in a Portuguese population.

Methods: This prospective study included 104 eyes. Detailed medical and ophthalmic examinations were performed; the PPCT was measured by spectral-domain optical coherence tomography (SD-OCT) using enhanced depth imaging (EDI) modality. The PPCT changes with MetS, as well as with other clinical and demographic factors, were investigated.

Results: The mean PPCT was $142.4 \pm 54.0 \mu \mathrm{m}(58-303 \mu \mathrm{m})$; it was thickest superiorly, followed by the temporal, nasal, and inferior sectors. The PPCT was significantly associated with axial length $(p<0.001)$, age $(p=0.001)$, intraocular pressure (IOP) $(p=0.041)$, weight $(p=0.015)$, and arterial hypertension $(p=0.044)$. The presence of MetS was associated with thinner PPCT in all sectors, being statistically significant in the temporal $(p=0.032)$ and inferotemporal $(p=0.034)$ sectors.

Conclusions: The choroidal thickness was significantly less in temporal and inferotemporal sectors in patients with MetS than in controls. This may suggest vascular insufficiency around the optic nerve head.
\end{abstract}

Keywords: Enhanced depth imaging, Metabolic syndrome, Optical coherence tomography, Peripapillary choroidal thickness

\section{Introduction}

The choroid is a vascularized and pigmented tissue that extends from the ora serrata to the optic nerve. It is supplied by the posterior ciliary arteries and the drainage is mainly through the vortex veins (1). This structure has the highest perfusion rate compared with any other vascular bed within the human body $(1,2)$. Therefore, it is rational to consider that metabolic disturbances, such as diabetes, arterial hypertension, and dyslipidemia, with documented implications in other highly irrigated organs (kidneys, heart, and brain), may also affect the choroid.

Accepted: November 3, 2016

Published online: November 21, 2016

Corresponding author:

Mónica Loureiro

R. Dr. Miguel Assuncao Lopes, n. 15, 3.B

Centro Hospitalar de V. N. Gaia/Espinho

4430-698 V.N. Gaia, Portugal

monicamloureiro@gmail.com
Enhanced depth imaging (EDI) for spectral-domain optical coherence tomography (SD-OCT) generates an inverted image by moving the choroid to the zero delay to maximize the sensitivity on the outer limit of choroid, making it possible to generate high-resolution images of posterior structures (3). Since EDI was described to visualize the choroid (3), studies have been focused on the measurement of its thickness, which depends on several factors. Thinner choroid has been associated with older age (4-6), higher intraocular pressure (IOP) (7), and longer axial length $(8,9)$.

The peripapillary choroid (PPC) has special interest for the study of optic nerve diseases, including glaucoma. The optic nerve head is primarily supplied by the PPC and the vascular theory attributes the development of glaucoma to an intraneural ischemia resulting from decreased perfusion of the optic nerve (10). In fact, a reduction in the average or regional PPC thickness (PPCT) has been reported in glaucoma (11-13). On the other hand, Rasoulinejad et al (14) showed an increased prevalence of components of metabolic syndrome in patients with glaucoma; however, the underlying mechanisms should be clarified.

The metabolic syndrome (MetS) is an important health problem worldwide (15); in Portugal, its prevalence adjusted 
for sex and age is high (27.5\%), rising progressively with increasing age ( $43.6 \%$ between 60 and 69 years of age) (15). According to the prevalence of MetS, it is important for ophthalmologists to establish a better conception of the association between MetS and eye diseases.

The aim of the present study was to investigate the impact of MetS on PPCT. In addition, we intended to characterize the PPCT in a Portuguese population.

\section{Methods}

\section{Study design and participants}

Subjects were recruited consecutively from the Ophthalmology Department of Centro Hospitalar de V.N. Gaia, Porto, Portugal. The study was approved by the local ethics committee and informed consent (according to the tenets of the Declaration of Helsinki) was obtained from participating patients.

The eligibility criteria were age $\geq 18$ years; no history of retinopathies, inflammatory eye disease, or optic neuropathy; absence of ocular surgery other than cataract surgery; absence of steroids use; and physical and mental ability to participate. Then, 2 groups were formed according to the status of MetS: patients with MetS and controls without MetS.

\section{Ophthalmic evaluation}

The ophthalmic evaluation consisted of best-corrected visual acuity, slit-lamp anterior and posterior segments examination, and Goldmann applanation tonometry. Corneal thickness using ultrasonic pachymetry (Ocuscan ${ }^{\circledR}$; Alcon, Fort Worth, TX, USA), axial length using interferometry (IOLMaster $^{\circledR}$; Carl Zeiss Meditec, Dublin, CA, USA), and PPCT using SD-OCT (Spectralis ${ }^{\circledR}$; Heidelberg Engineering, Heidelberg, Germany) were measured. To assess the PPC, we used EDI modality, the default glaucoma application, and the preset circular retinal nerve fiber layer scan, consisting of 100 averaged B-scans.

For the choroidal segmentation, the outer border was defined as the hyperreflective line of the inner surface of sclera or, if a clear line could not be detected, a smoothing line connecting the outer limits of the large choroidal vascular spaces was performed; the inner border was immediately below retinal pigment epithelium/Bruch membrane complex. Adopting the regional stratification included the temporal $(\mathrm{T})$ sector, superotemporal (ST) sector, superonasal (SN) sector, nasal (N) sector, inferonasal (IN) sector, and the inferotemporal (IT) sector, the average thickness of which was used for analysis.

\section{Metabolic syndrome evaluation}

The MetS was defined as the presence of 3 or more of the following (16):

1. Elevated fasting glucose $(\geq 100 \mathrm{mg} / \mathrm{dL})$

2. Elevated triglycerides $(\geq 150 \mathrm{mg} / \mathrm{dL}$ )

3. Reduced high-density lipoprotein $(<40 \mathrm{mg} / \mathrm{dL}$ in men and $<50 \mathrm{mg} / \mathrm{dL}$ in women)

4. Elevated blood pressure (BP) (systolic BP $\geq 130 \mathrm{~mm} \mathrm{Hg}$ and/or diastolic $\mathrm{BP} \geq 85 \mathrm{~mm} \mathrm{Hg}$ )
5. Abdominal obesity (waist circumference [WC] $\geq 94 \mathrm{~cm}$ for men and $\geq 80 \mathrm{~cm}$ for women)

Data referring to fasting glucose and lipid profile were collected from a national data program that shares patient information among health professionals of different health institutes (Plataforma de dados da Saúde [PDS $\left.{ }^{\circledR}\right]$ ). When not available or with more than 6 months, new blood tests were performed in the hospital. Resting BP was assessed 3 consecutive times on the upper arm using an automatic sphygmomanometer, and the mean value was used. The WC was measured at the midpoint between the lower border of the rib cage and the iliac crest while subjects were standing. Body weight and height were measured with the subjects barefoot and wearing light clothing.

\section{Statistical analysis}

The collected data were recorded and analyzed in SPSS ${ }^{\circledR}$ Statistics version 22.0 software (IBM, New York, NY, USA). One eye of each patient was randomly chosen for analysis.

The analysis consisted of descriptive estimates of the parameters evaluated as well as the evaluation of normality through Kolmogorov-Smirnov test. $t$ Tests were carried out to compare PPCT measurements across 2 independent groups. The univariate analysis, for continuous variable, was evaluated using linear regression between global PPCT (as dependent variable) and the different parameters as predictors. Finally, we carried out a multivariate regression analysis with the global PPCT (as dependent variable) and all of those variables as independent. In this step we use the selection procedure based on the Best Subsets Selection algorithm. The decision rule for all tests consists of detecting statistical evidence if the $p$ value of the test is less than 0.05 .

\section{Results}

We observed 104 eyes of 104 subjects; the mean age was $65 \pm 11.4$ years and $57.7 \%$ were women. The mean global PPCT was $142.4 \pm 54.0 \mu \mathrm{m}$ and ranged between 58 and $303 \mu \mathrm{m}$. The PPCT was thickest in the superior sectors (155.0 \pm $57.3 \mu \mathrm{m}$ in ST and $152.5 \pm 55.0 \mu \mathrm{m}$ in SN sectors), followed by T sector $(149.5 \pm 62.5 \mu \mathrm{m}), \mathrm{N}$ sector $(145.8 \pm 55.9 \mu \mathrm{m})$, and finally the inferior sectors (121.4 $\pm 52.3 \mu \mathrm{m}$ in IT and $120.7 \pm$ $50.9 \mu \mathrm{m}$ in IN sectors). The global PPCT did not statistically differ between men $(134.4 \pm 44.5 \mu \mathrm{m})$ and women $(148.3 \pm$ $59.8 \mu \mathrm{m}, \mathrm{p}=0.18$ ). There was a tendency for the global PPCT to decrease over the years and the age-related loss in PPCT took place in a similar amount in all sectors (Fig. 1).

In the univariate analysis (Tab. I), thicker global PPCT was significantly associated with younger age $(p<0.001$; standardized coefficient $\beta=-0.40$ ), and was related with the systemic parameter of lower fasting glucose $(p=0.04 ; \beta=-0.21)$ and with the ocular parameter of shorter axial length $(p<0.001$; $\beta=-0.41$ ). For nominal variables (components of MetS), we performed a comparison test of the mean global PPCT (Tab. II) and there was a statistically significant association with arterial hypertension $(p=0.01)$. In the multivariate analysis (Tab. III), thicker PPCT was significantly associated with shorter axial length $(p<0.001)$, younger age $(p=0.001)$, 

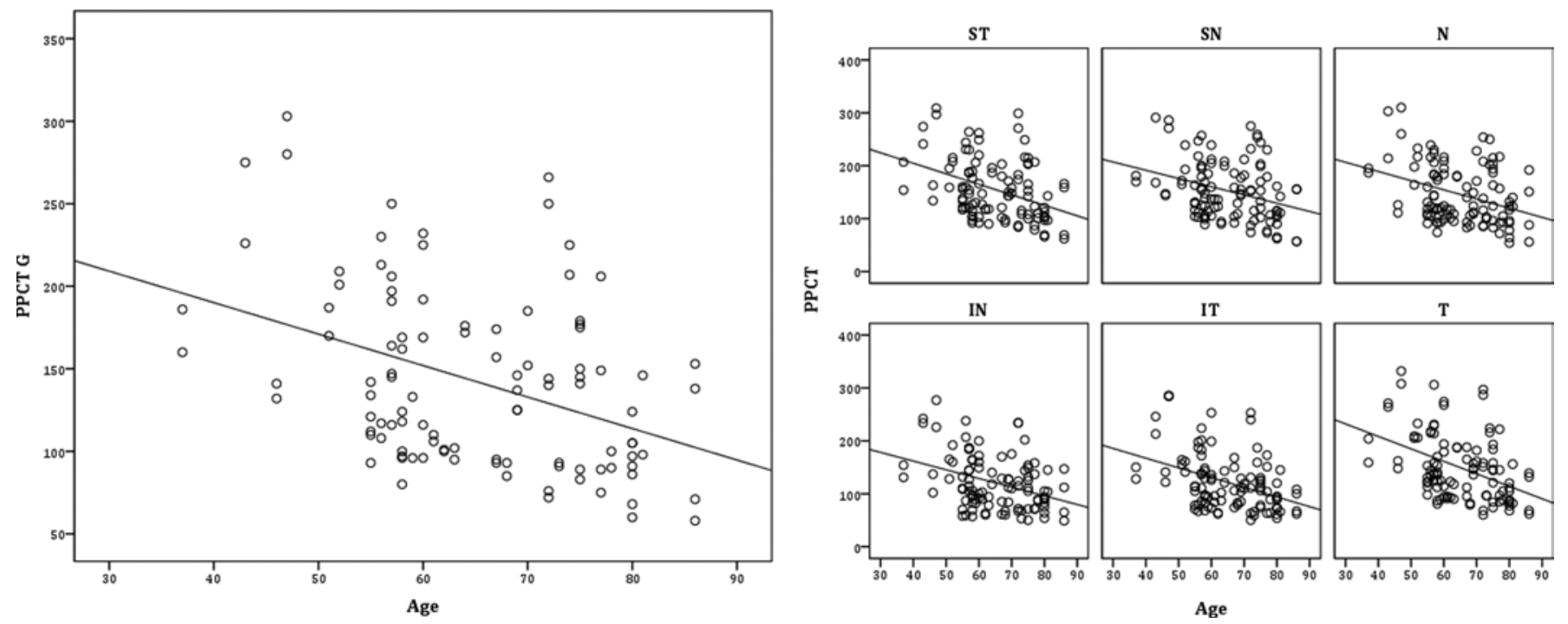

Fig. 1 - Bivariate scatterplots of global and sectorial peripapillary choroidal thickness (PPCT) ( $\mu \mathrm{m})$ versus age (years). IN =inferonasal sector; $\mathrm{IT}=$ inferotemporal sector; $\mathrm{N}=$ nasal sector; $\mathrm{SN}=$ superonasal sector; $\mathrm{ST}=$ superotemporal sector; $\mathrm{T}$ = temporal sector.

TABLE I - Univariate regression analysis with dependent variable global peripapillary choroidal thickness

\begin{tabular}{|c|c|c|c|c|c|}
\hline \multirow[t]{2}{*}{ Parameters } & \multirow[t]{2}{*}{$p$ value } & \multirow{2}{*}{$\begin{array}{l}\text { Regression } \\
\text { coefficient, } \beta\end{array}$} & \multirow{2}{*}{$\begin{array}{c}\text { Standardized } \\
\text { coefficient }\end{array}$} & \multicolumn{2}{|c|}{$95 \% \mathrm{Cl}$ for $\beta$} \\
\hline & & & & Lower limit & Upper limit \\
\hline Age & $<0.001^{\mathrm{a}}$ & -1.907 & -0.400 & -2.764 & -1.050 \\
\hline Body mass index & 0.799 & 0.263 & 0.025 & -1.776 & 2.301 \\
\hline Systolic BP, mm Hg & 0.063 & -0.683 & -0.183 & -1.403 & 0.038 \\
\hline Diastolic BP, mm Hg & 0.898 & -0.067 & -0.013 & -1.102 & 0.968 \\
\hline $\mathrm{LDL}, \mathrm{mmol} / \mathrm{L}$ & 0.058 & 0.328 & 0.187 & -0.011 & 0.667 \\
\hline Triglycerides, mmol/L & 0.432 & -0.093 & -0.078 & -0.326 & 0.140 \\
\hline Cholesterol, mmol/L & 0.073 & 0.280 & 0.176 & -0.027 & 0.588 \\
\hline Fasting glucose, $\mathrm{mmol} / \mathrm{L}$ & $0.037^{a}$ & -0.647 & -0.207 & -1.253 & -0.040 \\
\hline Axial length, mm & $<0.001^{\mathrm{a}}$ & -24.556 & -0.406 & -35.420 & -13.691 \\
\hline $\mathrm{CCT}, \mu \mathrm{m}$ & 0.942 & 0.012 & 0.007 & -0.303 & 0.327 \\
\hline
\end{tabular}

a Significant for 0.05 level.

$\mathrm{BCVA}=$ best-corrected visual acuity; $\mathrm{BP}=$ blood pressure; $\mathrm{CCT}=$ central corneal thickness; $\mathrm{Cl}=$ confidence interval; $\mathrm{HDL}=$ high-density lipoprotein; $\mathrm{LDL}=$ low-density lipoprotein; IOP = intraocular pressure; $W C$ = waist circumference.

TABLE II - Independent sample $t$ test results

\begin{tabular}{lccc}
\hline Parameters & p value & \multicolumn{2}{c}{$\mathbf{9 5 \%} \mathbf{C l}$ for $\boldsymbol{\beta}$} \\
\cline { 3 - 4 } & & Lower limit & Upper limit \\
\hline Arterial hypertension & $0.007^{\mathrm{a}}$ & 10.10 & 59.45 \\
Reduced HDL & 0.081 & -2.28 & 37.99 \\
Elevated triglycerides & 0.448 & -12.08 & 26.82 \\
Elevated fasting glucose & 0.522 & -14.80 & 28.99 \\
Elevated waist & 0.514 & -34.10 & 64.61 \\
circumference & & & \\
\hline
\end{tabular}

a Significant for 0.05 level.

$\mathrm{Cl}=$ confidence interval; $\mathrm{HDL}=$ high-density lipoprotein. higher weight $(p=0.02)$, lower IOP $(p=0.04)$, and absence of arterial hypertension $(p=0.04)$.

Forty-four subjects (42.3\%) had MetS (patients group) and 60 subjects $(57.7 \%)$ did not present MetS (controls). In the MetS group, the mean age was $66.9 \pm 11.6$ years, the mean IOP was $15.1 \pm 2.0 \mathrm{~mm} \mathrm{Hg}$, the mean axial length was $23.1 \pm 0.9 \mathrm{~mm}$, and the mean corneal thickness was $538.7 \pm$ $30.6 \mu \mathrm{m}$; the corresponding figures in the control group were $63.6 \pm 11.1(p=0.14), 14.7 \pm 2.2(p=0.43), 23.0 \pm 0.9(p=$ $0.74)$, and $531.8 \pm 35.8(p=0.31)$. The mean global PPCT in the MetS group was $133.2 \pm 39.4 \mu \mathrm{m}$ and in the control group was $148.2 \pm 63.4 \mu \mathrm{m}$; there was no significant difference between the 2 groups $(p=0.17)$. Analyzing by sectors, the 
TABLE III - Multivariate analysis with dependent variable global peripapillary choroidal thickness

\begin{tabular}{|c|c|c|c|c|c|c|}
\hline & \multirow[t]{2}{*}{ Unstandardized coefficients, $\beta$} & \multirow[t]{2}{*}{ p value } & \multicolumn{2}{|c|}{$95.0 \% \mathrm{Cl} \beta$} & \multirow[t]{2}{*}{ Importance } & \multirow[t]{2}{*}{ VIF } \\
\hline & & & Lower bound & Upper bound & & \\
\hline Axial length & -25.597 & $<0.001^{\mathrm{a}}$ & -35.606 & -15.587 & 0.466 & 1.075 \\
\hline Body weight & 0.743 & $0.015^{\mathrm{a}}$ & 0.150 & 1.336 & 0.112 & 1.139 \\
\hline IOP & -4.244 & $0.041^{a}$ & -8.303 & -0.185 & 0.078 & 1.122 \\
\hline
\end{tabular}

${ }^{a}$ Significant for 0.05 level.

$\mathrm{Cl}$ = confidence interval; IOP = intraocular pressure; $\mathrm{VIF}=$ Variance Inflation Factor.

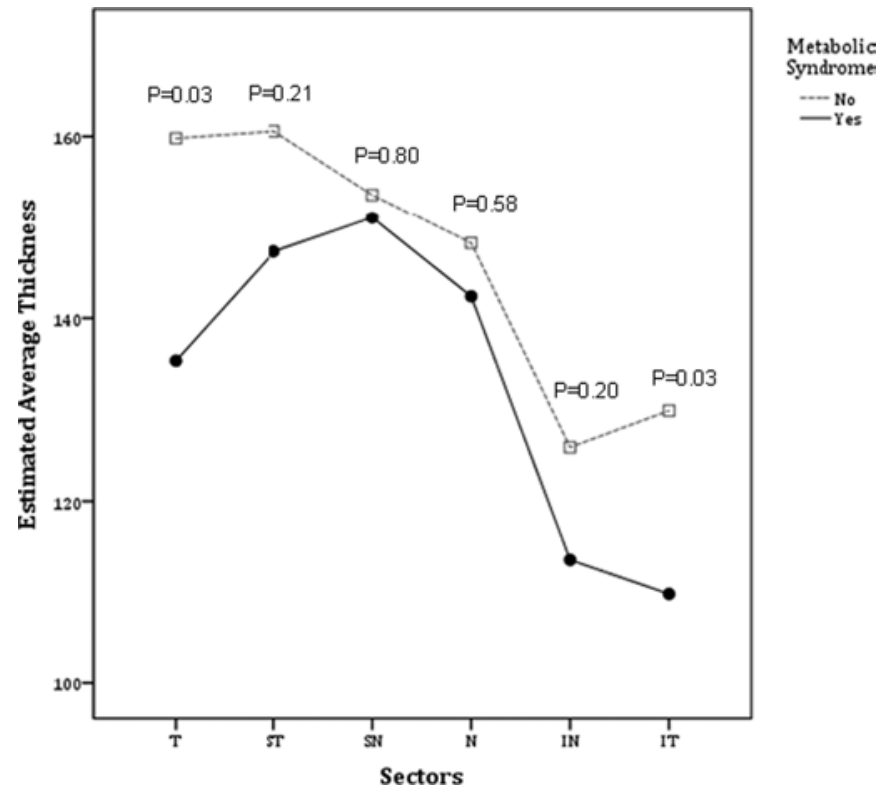

Fig. 2 - Profile charts of peripapillary choroidal thickness $(\mu \mathrm{m})$ by sectors in the presence and absence of metabolic syndrome. IN = inferonasal sector; IT = inferotemporal sector; $\mathrm{N}=$ nasal sector; $\mathrm{SN}=$ superonasal sector; $\mathrm{ST}=$ superotemporal sector; $\mathrm{T}=$ temporal sector.

presence of MetS was associated with thinner choroid in all sectors and the difference was statistically significant in the T $(p=0.03)$ and IT sectors ( $p=0.03)$ (Fig. 2).

\section{Discussion}

In our study, the global PPCT was $142.4 \pm 54.0 \mu \mathrm{m}$; to our knowledge, this was the first evaluation of PPCT in Portuguese subjects. Previous studies have reported mean PPCT between 134 and $191 \mu \mathrm{m}(8,11,17,18)$; the disparities found in the literature may be due to differences in the demographic data of enrolled subjects such as ethnicity and age. In the Huang et al (18) study, for example, the thickness was considerably greater but the mean age of the participants was 48 years, vs the 65 years of our study.

The PPCT was thickest superiorly and thinnest inferiorly, while in between, it was thicker in the temporal sector than in the nasal sector; the regional differences that we verified are in agreement with other published data $(8,18,19)$. Although this asymmetric distribution shows a characteristic pattern, the reason remains unknown.

A decrease of the global PPCT was shown over the years; with each year increase in age, the global PPCT decreased by about $2 \mu \mathrm{m}$ (Tab. I). The age-related decrease was identical to what is already described $(8,11,12)$. With every axial elongation of $1 \mathrm{~mm}$, the global PPCT decreased $24.6 \mu \mathrm{m}$ (Tab. I). In a similar manner, Roberts et al (11) reported a decline of $10 \mu \mathrm{m}$ with each $\mathrm{mm}$ more of axial length. The underlying mechanism is probably related to the elongation of the eyeball, which leads to mechanical stretching and thinning of the retina and choroid.

The PPCT was associated with IOP, age, and axial length, as previously described (4-9). It was also associated with arterial hypertension, which may be explained by the effect of the high intravascular pressure in the vessels, promoting sclerosis and contraction (20). The association between thicker PPCT and higher weight should be confirmed in other studies; the increased circulation volume in these patients can help to partially explain it.

The prevalence of MetS adjusted for age in Portugal is high (15), and is increasing related to sedentary habits and unbalanced diets; therefore we already expected that more than $40 \%$ of subjects would have MetS. In the presence of MetS, the PPC was significantly thinner in TI and T sectors. The macrovascular and microvascular impact of the MetS is well-documented in several organs (15), and may also influence the choriocapillaris architecture and the blood supply in these choroidal sectors.

Reduced PPCT results in a reduced ability to provide sufficient levels of oxygen and other metabolites to the optic nerve head, and as early as 1928, Elsching suggested that a compromise of the choroidal supply to the peripapillary area may be an etiologic factor in glaucoma (12). The thinnest sectors in the presence of MetS may represent areas of lower blood supply, which may predispose the optic nerve to glaucomatous ischemic damage $(17,19)$. This may be an underlying mechanism of the increased prevalence of MetS in patients with glaucoma (14).

Potential limitations of our study should be mentioned. First, we considered $p$ value less than 0.05 to be statistically significant, although as we performed multiple $t$ tests a $p$ value less than 0.01 could be more accurate. Second, there is lack of automatic segmentation of the choroid; however, previous studies have shown the reproducibility of manual 
segmentation (10), and the EDI permitted us to clearly see the boundaries of the choroid in all scans. Diurnal (circadian) variation of about 20 to $30 \mu \mathrm{m}$ change in choroidal thickness has been reported $(21,22)$; however, as the measurement was performed during 4:30-6:30 PM, it is unlikely that dependence of choroidal thickness on time of examination introduced a bias into our study.

\section{Conclusion}

In Portuguese subjects, the mean PPCT was $142.4 \pm$ $54.0 \mu \mathrm{m}$. Thicker choroid was significantly associated with shorter axial length, younger age, lower IOP, higher weight, and absence of arterial hypertension.

There may be an association between the presence of MetS and reduced PPCT in T and IT sectors, which may represent anatomic evidence of vascular compromise around the optic nerve head, related to MetS, and support the recommendation that patients with MetS should undergo regular ophthalmologic examinations.

\section{Disclosures}

Financial support: Partially supported by a grant of the Portuguese Society of Ophthalmology.

Conflict of interest: None of the authors has conflict of interest with this submission.

\section{References}

1. Kaufman PL, Alm A. Anatomy of the ocular circulation. Adler's physiology of the eye. $10^{\text {th }}$ ed. St. Louis: Elsevier Health Sciences; 2002.

2. Nickla DL, Wallman J. The multifunctional choroid. Prog Retin Eye Res. 2010;29(2):144-168.

3. Spaide RF, Koizumi H, Pozzoni MC. Enhanced depth imaging spectral-domain optical coherence tomography [erratum 2009;148:325]. Am J Ophthalmol. 2008;146(4):496-500.

4. Margolis R, Spaide RF. A pilot study of enhanced depth imaging optical coherence tomography of the choroid in normal eyes. Am J Ophthalmol. 2009;147(5):811-815.

5. Spaide RF. Age-related choroidal atrophy. Am J Ophthalmol. 2009;147(5):801-810.

6. Ramrattan RS, van der Schaft TL, Mooy CM, de Bruijn WC, Mulder PG, de Jong PT. Morphometric analysis of Bruchs membrane, the choriocapillaris, and the choroid in aging. Invest Ophthalmol Vis Sci. 1994;35(6):2857-2864.

7. Arora KS, Jefferys JL, Maul EA, Quigley HA. The choroid is thicker in angle closure than in open angle and control eyes. Invest Ophthalmol Vis Sci. 2012;53(12):7813-7818.

8. Jiang R, Wang YX, Wei WB, Xu L, Jonas JB. XU L, Jonas JB. Peripapillary choroidal thickness in adult Chinese: the Beijing Eye Study. Invest Ophthalmol Vis Sci. 2015;56(6):4045-4052.
9. Lee SW, Yu SY, Seo KH, Kim ES, Kwak HW. Diurnal variation in choroidal thickness in relation to sex, axial length, and baseline choroidal thickness in healthy Korean subjects. Retina. 2014;34(2):385-393.

10. Banitt M. The choroid in glaucoma. Curr Opin Ophthalmol. 2013;24(2):125-129.

11. Roberts KF, Artes PH, OLeary N, et al. Peripapillary choroidal thickness in healthy controls and patients with focal, diffuse, and sclerotic glaucomatous optic disc damage. Arch Ophthalmol. 2012;130(8):980-986.

12. Park HY, Lee NY, Shin HY, Park CK. Analysis of macular and peripapillary choroidal thickness in glaucoma patients by enhanced depth imaging optical coherence tomography. J Glaucoma. 2014;23(4):225-231.

13. Hirooka K, Tenkumo K, Fujiwara A, Baba T, Sato S, Shiraga F. Evaluation of peripapillary choroidal thickness in patients with normal-tension glaucoma. BMC Ophthalmol. 2012;12:29.

14. Rasoulinejad SA, Kasiri A, Montazeri M, et al. The association between primary open angle glaucoma and clustered components of metabolic syndrome. Open Ophthalmol J. 2015;9:149-155.

15. Fiuza M, Cortez-Dias N, Martins S, Belo A; VALSIM study investigators. Metabolic syndrome in Portugal: prevalence and implications for cardiovascular riskresults from the VALSIM Study. Rev Port Cardiol. 2008;27(12):1495-1529.

16. Alberti KG, Eckel RH, Grundy SM, et al; International Diabetes Federation Task Force on Epidemiology and Prevention; National Heart, Lung, and Blood Institute; American Heart Association; World Heart Federation; International Atherosclerosis Society; International Association for the Study of Obesity. Harmonizing the metabolic syndrome: a joint interim statement of the International Diabetes Federation Task Force on Epidemiology and Prevention; National Heart, Lung, and Blood Institute; American Heart Association; World Heart Federation; International Atherosclerosis Society; and International Association for the Study of Obesity. Circulation. 2009;120(16):1640-1645.

17. Gupta $P$, Jing $T$, Marziliano $P$, et al. Peripapillary choroidal thickness assessed using automated choroidal segmentation software in an Asian population. Br J Ophthalmol. 2015;99(7):920-926.

18. Huang $W$, Wang $W$, Zhou $M$, et al. Peripapillary choroidal thickness in healthy Chinese subjects. BMC Ophthalmol. 2013;13:23.

19. Ho J, Branchini L, Regatieri C, Krishnan C, Fujimoto JG, Duker JS Analysis of normal peripapillary choroidal thickness via spectral domain optical coherence tomography. Ophthalmology. 2011;118(10):2001-2007.

20. Akay F, Gundogan FC, Yolcu U, Toyran S, Uzun S. Choroidal thickness in systemic arterial hypertension. Eur J Ophthalmol. 2016;26(2):152-157.

21. Tan CS, Ouyang Y, Ruiz H, Sadda SR. Diurnal variation of choroidal thickness in normal, healthy subjects measured by spectral domain optical coherence tomography. Invest Ophthalmol Vis Sci. 2012;53(1):261-266.

22. Usui $S$, Ikuno $Y$, Akiba $M$, et al. Circadian changes in subfoveal choroidal thickness and the relationship with circulatory factors in healthy subjects. Invest Ophthalmol Vis Sci. 2012;53(4):2300-2307. 